\title{
Besearch S Surare \\ Discussion on the damping factor and improvement of the Levenberg-Marquardt method
}

\section{Wenwu Zhu ( $\nabla$ fmccea@sina.com )}

The First Crust Monitoring and Application Centre, China Earthquake Administration, Tianjin 300180, China

\section{Article}

Keywords: Gravity inversion, Levenberg-Marquardt method, damping factor

Posted Date: October 19th, 2021

DOI: https://doi.org/10.21203/rs.3.rs-985868/v1

License: (c) (i) This work is licensed under a Creative Commons Attribution 4.0 International License. Read Full License 
Discussion on the damping factor and improvement of the Levenberg-Marquardt method

\section{Abstract}

The ill-posed problem is the key obstacle to obtain the accurate inversion results in the geophysical inversion field, and the Levenberg-Marquardt ${ }^{1,2}$ (hereinafter referred to as the L-M method) method has been widely used as it can effectively improve the ill-posed problems. However, the inversion results obtained by the L-M method are usually stable but incorrect, the reason is that the damping factor in the L-M method is difficult to solve, and it is usually approximated with a positive constant by experience or through some fitting methods. This paper uses the binary gravity model to demonstrate that the damping factor in the L-M method cannot be regarded as a positive constant only, it should have the following characteristics: (i) the damping factor is a vector, not just a constant; (ii) the values of the vector are composed of both positive and negative constants, not just positive constants; (iii) the corresponding value in the vector is close or equal to $\infty$ when the corresponding density block's value is close or equal to zero. Even if the above characteristics have been found in the L-M method, it is difficult to reasonably estimate the damping factor as the damping factor oscillate severely due to the third characteristic, and the improved L-M method is proposed which effectively avoids the damping factor's severe oscillation problem. The strategy of obtaining the reasonable damping factor is given finally.

\section{Key words}

Gravity inversion; Levenberg-Marquardt method; damping factor

\section{Introduction}

The significance of the gravity inversion study for exploring the underground density structure is self-evident. Generally, the inversion models are usually simplified before implementing the gravity inversion study, and the binary or ternary inversion models have received widespread attention ${ }^{3,4,5,6,7,8,9,10,11,12}$. However, even if we implement our inversion research on the binary or ternary model, the inversion results are usually not ideal because they are severely affected by the ill-posed problems which usually appear during the gravity inversion calculations. Most scholars try to solve the ill-posed problems based on the L-M method which is also called the Regularization method ${ }^{13}$ (the L-M inversion equation is constructed by the residual term, the 
damping term and the damping factor, which are equivalent to the residual term, the Regularization term and the Regularization factor in the Regularization equation, respectively). In other words, the L-M method are the foundation of the geophysical inversion field and most of the new inversion methods are derived from the L-M method. The ill-posed problems can be well solved by the L-M method, but the inversion results are usually stable but not correct. The key reason is that the damping factor of the L-M method is always considered to be a positive constant! (Some methods derived from the L-M method indeed obtain ideal inversion results but these methods are established on adding additional constraints which are often difficult to obtain in practical applications or promote due to their particularities $7,14,15,16,17,18$. This paper does not consider the situation of adding additional constraints, and only the unique constraint of the L-M method is further processed and used for the Binary inversion model in this paper).

This paper is devoted to demonstrate why the damping factor of the L-M method cannot be just a positive constant, summarizing the characteristics of the damping factor of the L-M method, and improving the L-M method with almost no additional constraints.

\section{Discussion of the damping factor of the L-M method}

Analysis of the principle of the damping factor of the L-M method

This paper takes the 2.5-dimensional (2.5D) binary inversion model as an example. Fig.1 represents a profile of the $2.5 \mathrm{D}$ binary model composed by the density blocks $m(\mathrm{q} \times 1)$, and the corresponding gravity anomaly data containing the Gaussian noise is $\tilde{d}(p \times 1)$. The kernel function matrix is $G(p \times q)$. Apparently, we have:

$$
G \widetilde{\boldsymbol{m}}=\widetilde{\boldsymbol{d}} .
$$

$\widetilde{m}$ represents the approximation of the real density value $m$. 


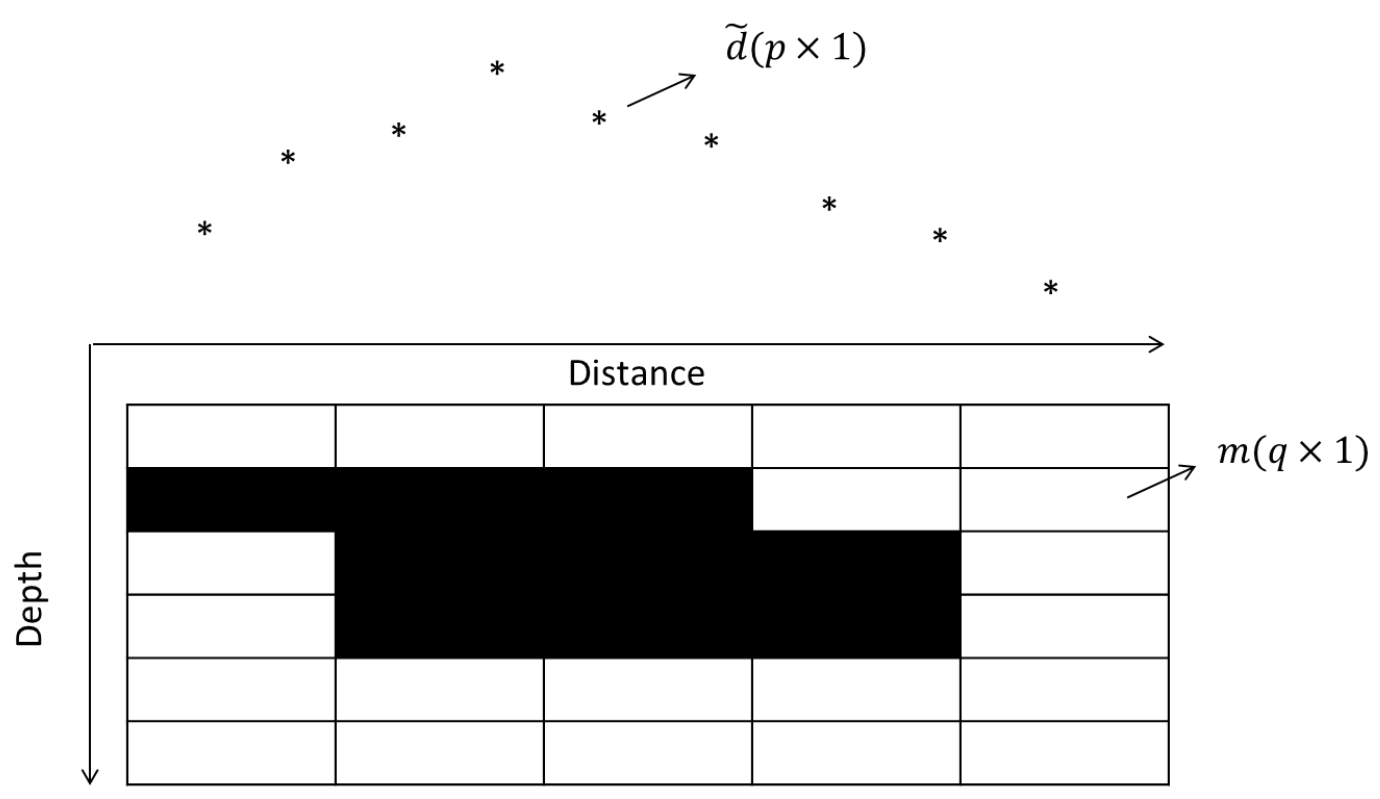

Fig.1 Illustration of the 2.5D Binary inversion model composed by the density blocks $m(q \times 1)$ and the corresponding gravity anomaly data $\tilde{d}(\mathrm{p} \times 1)$

If the number of the gravity data is greater than the number of the unknown density blocks $(p>q)$ and the kernel matrix G is not ill-posed, we only need to use the simple Least Squares method to easily obtain the ideal inversion results:

$$
\operatorname{Minimize}\left\|W_{d}(G \tilde{m}-\widetilde{d})\right\|_{2}^{2} \text {. }
$$

$W_{d}$ represents the weighting function of the gravity anomaly data $\tilde{d}$.

After differentiating the above formula, we can obtain:

$$
\widetilde{\boldsymbol{m}}=\left(\mathbf{G}^{\mathbf{T}} \mathbf{W}_{\mathbf{d}}{ }^{\mathbf{T}} \mathbf{W}_{\mathbf{d}} \mathbf{G}\right)^{-\mathbf{1}} \mathbf{G}^{\mathbf{T}} \mathbf{W}_{\mathbf{d}}{ }^{\mathbf{T}} \widetilde{\boldsymbol{d}} .
$$

In equation (3), ${ }^{\mathrm{T}}$ is the transpose of $\cdot$

However, the kernel matrix G is usually ill-posed so that we cannot simply apply equation (3) to the inversion calculations (Generally, G is well-posed only if the number of the density blocks is extremely limited).

Fortunately, there is always some additional geophysical information which can be easily obtained without wasting too much manpower and financial resources in the geophysical inversion field, and the information can be applied to the inversion calculation so that the inversion solution can be constrained in a more reasonable or compact range. For example, the values of the density blocks must be within a certain range (e.g. greater than zero and less than infinity at least, and cannot 
be any real numbers) for the underground substances, then we can easily obtain the following constraint equation:

$$
\|\widetilde{\boldsymbol{m}}\|=\mathbf{k}, \mathbf{k} \geq \mathbf{0} .
$$

This constraint is also applied to the Binary inversion model for which there are only two kinds of values in the inversion model.

And combined with equation (4), Marquardt give the following well-known L-M inversion equation based on equation (2):

$$
\begin{gathered}
\text { Minimize }\left\|\mathbf{W}_{\mathbf{d}}(\mathbf{G} \widetilde{\boldsymbol{m}}-\widetilde{\boldsymbol{d}})\right\|_{2}^{2} \\
\text { s.t. }\|\widetilde{\boldsymbol{m}}\|=\mathbf{k}, \mathbf{k} \geq \mathbf{0}
\end{gathered}
$$

In equation (5), $\mathrm{k}$ is a positive constant which does not need to be known in the inversion calculation process. According to equation (5), using the Lagrange Multiplier Method, we can obtain:

$$
G^{T} \mathbf{W}_{d}^{T} \mathbf{W}_{d} \mathbf{G} \widetilde{m}+\lambda_{0} \widetilde{m}=G^{T} \mathbf{W}_{d}^{T} \mathbf{W}_{d} \widetilde{d}
$$

Furthermore, we can obtain:

$$
\widetilde{\boldsymbol{m}}=\left(\mathbf{G}^{\mathrm{T}} \mathbf{W}_{\mathrm{d}}^{\mathrm{T}} \mathbf{W}_{\mathrm{d}} \mathbf{G}+\boldsymbol{\lambda}_{\mathbf{0}} \mathbf{I}\right)^{-1} \mathbf{G}^{\mathrm{T}} \mathbf{W}_{\mathbf{d}}^{\mathrm{T}} \mathbf{W}_{\mathrm{d}} \widetilde{\boldsymbol{d}}
$$

Here, $\lambda_{0}$ is regarded as a positive constant ${ }^{1,2}$, I is the identity matrix. Comparing with equation (3), the positive constant $\lambda_{0}$ is added to the diagonal of $G^{T} W_{d}{ }^{T} W_{d} G$ in equation (7), which makes the condition number of $\mathrm{G}^{\mathrm{T}} \mathrm{W}_{\mathrm{d}}{ }^{\mathrm{T}} \mathrm{W}_{\mathrm{d}} \mathrm{G}$ improved and the inversion solution stable.

Although the L-M method makes the inversion solution stable, the inversion solution is usually incorrect which is greatly different from the actual density value. The specific analysis is as follows:

Assuming the real gravity data and the corresponding noise are $d$ and $\Delta d$, respectively, and the real values of the density blocks and the corresponding residual of the inversion results caused by $\Delta d$ are $m$ and $\Delta m$, respectively, which is:

$$
\begin{gathered}
\widetilde{\boldsymbol{d}}=\boldsymbol{d}+\Delta \boldsymbol{d}, \\
\widetilde{\boldsymbol{m}}=\boldsymbol{m}+\Delta \boldsymbol{m} .
\end{gathered}
$$

Now substitute equation (8) into equation (6) (Let $\mathrm{W}=\mathrm{W}_{\mathrm{d}}{ }^{\mathrm{T}} \mathrm{W}_{\mathrm{d}}$ in equation (6) for ease of writing): 


$$
\mathbf{G}^{\mathrm{T}} \mathbf{W G}(\boldsymbol{m}+\Delta \boldsymbol{m})+\lambda_{\mathbf{0}}(\boldsymbol{m}+\Delta \boldsymbol{m})=\mathbf{G}^{\mathrm{T}} \mathbf{W}(\boldsymbol{d}+\Delta d)
$$

Because $\mathrm{Gm}=d$, and we have :

$$
\mathbf{G}^{\mathrm{T}} \mathbf{W G m}=\mathbf{G}^{\mathrm{T}} \mathbf{W d}
$$

Now substitute equation (10) into equation (9):

$$
\mathbf{G}^{\mathrm{T}} \mathbf{W G} \Delta \boldsymbol{m}+\boldsymbol{\lambda}_{\mathbf{0}}(\boldsymbol{m}+\Delta \boldsymbol{m})=\mathbf{G}^{\mathrm{T}} \mathbf{W} \Delta d
$$

Apparently, we hope $\widetilde{m}=m+\Delta m \rightarrow m$ or $\Delta m \rightarrow 0$, then

$$
\begin{gathered}
\mathbf{G}^{\mathrm{T}} \mathbf{W G} \Delta \boldsymbol{m} \rightarrow \mathbf{0} \\
\lambda_{\mathbf{0}} \Delta \boldsymbol{m} \rightarrow \mathbf{0}
\end{gathered}
$$

Now substitute equation (12) into equation (11):

$$
\lambda_{0} I m \approx \mathbf{G}^{\mathrm{T}} \mathbf{W} \Delta d
$$

According to equation (13), the accurate $\lambda_{0}$ can be obtained if the real density value $m$ and the noise of the gravity data $\Delta d$ is known. Now the specific form of the $i$ th expression in equation (13) is given as follows:

$$
\lambda_{0} m_{i} \approx \mathrm{G}^{\mathrm{T}}{ }_{i} \mathrm{~W} \Delta d, \mathbf{i}=1,2,3, \ldots, \mathrm{q}
$$

In equation (14), $m_{i}$ represents the $i$ th value of the real values $m, \mathrm{G}^{\mathrm{T}}{ }_{i}$ represents the $i$ th row of $G^{T}$.

Then we give two conclusions as follows based on equation (14):

i) $\lambda_{0}$ in the L-M method should be replaced by a vector $\lambda\left(\lambda=\left[\lambda_{1}, \lambda_{2}, \ldots, \lambda_{\mathrm{q}}\right]^{\mathrm{T}}\right)$.

Analysis: if $\mathrm{m}_{\mathrm{i}} \neq 0$, according to equation (14), for each $\mathrm{m}_{\mathrm{i}}$ :

$$
\lambda_{0} \approx \frac{\mathrm{G}^{\mathrm{T}}{ }_{i} \mathrm{~W} \Delta d}{m_{\mathrm{i}}}, m_{\mathrm{i}} \neq \mathbf{0} \mathrm{i}=1,2,3, \ldots \mathrm{q}
$$

And according to the L-M method, $\lambda_{0}$ is a constant, so the following expression should always hold:

$$
\frac{\mathrm{G}^{\mathrm{T}}{ }_{\mathrm{i}} \mathrm{W} \Delta d}{m_{i}} \approx \frac{\mathrm{G}^{\mathrm{T}}{ }_{\mathrm{j}} \mathrm{W} \Delta d}{m_{j}}, m_{\mathrm{i}}, m_{\mathrm{j}} \neq \mathbf{0} \mathbf{i}, \mathbf{j}=1,2,3, \ldots \mathbf{q}
$$

However, we know that $\mathrm{G}_{\mathrm{i}}^{\mathrm{T}} \neq \mathrm{G}^{\mathrm{T}}{ }_{\mathrm{j}}$ due to the characteristics of $\mathrm{G}$, and apparently $\mathrm{m}_{\mathrm{i}}$ is probably not equal to $\mathrm{m}_{\mathrm{j}}$, so $\frac{\mathrm{G}^{\mathrm{T}}{ }_{\mathrm{i}} \mathrm{W} \Delta d}{\mathrm{~m}_{\mathrm{i}}}$ is probably not equal to $\frac{\mathrm{G}^{\mathrm{T}}{ }_{\mathrm{j}} \mathrm{W} \Delta d}{\mathrm{~m}_{\mathrm{j}}}$ in equation (16). Therefore, 
equation (16) cannot always hold, which means we cannot just use a constant $\lambda_{0}$ to represent all the $\frac{\mathrm{G}_{\mathrm{T}}^{\mathrm{T}} \mathrm{W} \Delta d}{\mathrm{~m}_{\mathrm{i}}}$ shown in equation (15). Instead, we should use a series of constants which construct a new vector $\lambda$ to represent $\frac{\mathrm{G}^{\mathrm{T}} \mathrm{W} \Delta d}{\mathrm{~m}_{\mathrm{i}}}$ not just a constant $\lambda_{0}$, and the values in the vector $\lambda$ may be either positive and negative which depends on whether $\mathrm{G}^{\mathrm{T}}{ }_{\mathrm{i}} \mathrm{W} \Delta d$ and $\mathrm{m}_{\mathrm{i}}$ are positive or negative.

The specific expression form of the vector $\lambda$ is as follows:

$$
\begin{gathered}
\lambda=\left[\lambda_{1}, \lambda_{2}, \ldots, \lambda_{q}\right]^{\mathbf{T}}, \\
\text { and } \lambda_{i} m_{\mathbf{i}} \approx \mathbf{G}^{\mathrm{T}}{ }_{\mathbf{i}} \mathbf{W} \Delta d, \mathbf{i}=\mathbf{1}, \mathbf{2}, \mathbf{3}, \ldots \mathbf{q} \text {. }
\end{gathered}
$$

So, the L-M method shown in equation (7) should be modified into the following form:

$$
\begin{gathered}
\widetilde{\boldsymbol{m}}=\left(\mathbf{G}^{\mathrm{T}} \mathbf{W G}+\operatorname{diag}(\lambda)\right)^{-1} \mathbf{G}^{\mathrm{T}} \mathbf{W} \widetilde{\boldsymbol{d}}, \\
\lambda=\left[\lambda_{1}, \lambda_{2}, \ldots, \lambda_{q}\right]^{\mathrm{T}} .
\end{gathered}
$$

$\operatorname{diag}(\lambda)$ represents a diagonal matrix with the elements of the vector $\lambda$.

ii) if $m_{i} \rightarrow 0$ or $=0$ and $\mathrm{G}^{\mathrm{T}}{ }_{\mathrm{i}} \mathrm{W} \Delta d \neq 0, \lambda_{\mathrm{i}} \rightarrow \infty$ or $=\infty$.

Analysis: This conclusion is obvious according to equation (17), the value of $\lambda_{i}$ will be approaching or equal to $\infty$ when the corresponding density value $m_{i} \rightarrow 0$ or $=0$. Because when $\mathrm{G}_{\mathrm{i}}^{\mathrm{T}} \mathrm{W} \Delta d \neq 0, \lambda_{i}$ needs to play a balanced role to assure equation (17) holds.

Synthetic test on the damping factor of the L-M method

We give a specific synthetic test to verify the correctness of the discussion on the damping factor of the L-M method above. In Fig.2a, the gravity dataset is obtained by forward modelling, in which the red dots represent the real values of the gravity dataset and the black line represents the contaminated gravity dataset by Gaussian noise equivalent to $5 \%$ of the accurate datum. And in Fig. $2 \mathrm{~b}$, the corresponding density blocks ( 5 rows $\times 6$ columns $=30$ blocks in total) are composed of the same square with a length of $100 \mathrm{~m}$, the black rectangles represent the density blocks with a value of $1 \mathrm{~kg} / \mathrm{m}^{3}$ and the other white areas represent the values of the density blocks are $0 \mathrm{~kg} / \mathrm{m}^{3}$. It is difficult to obtain ideal inversion results for this kind of the density model due to the severe overlap in the vertical direction and the hollow interior. 

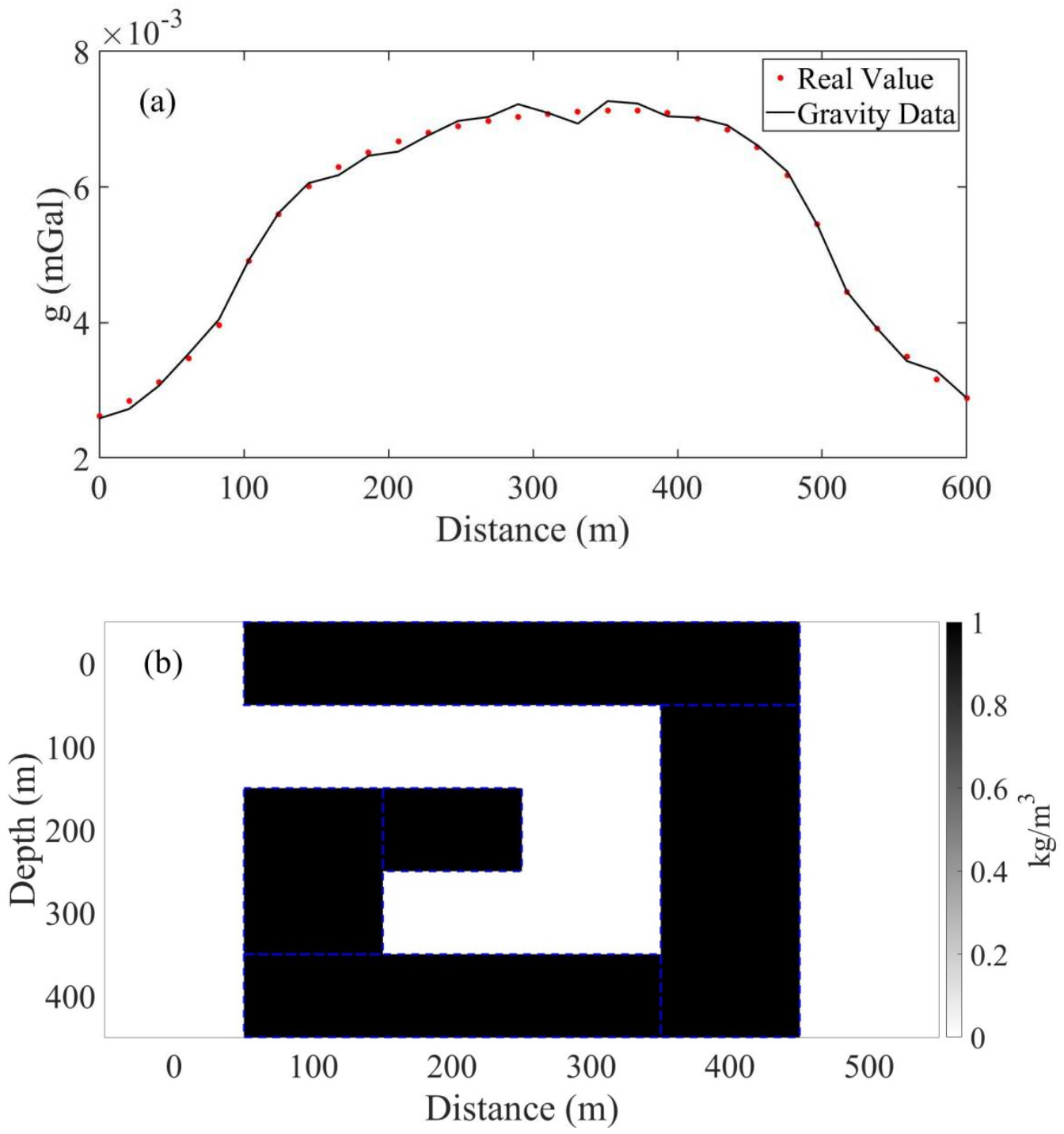

Fig.2 The gravity dataset and the corresponding density model. a-b, The real gravity dataset (red dots) and the contaminated gravity dataset (black line) shown in (a), the density model is composed of the black blocks with a value of $1 \mathrm{~kg} / \mathrm{m}^{3}$ and the white areas with with a value of 0

$$
\mathrm{kg} / \mathrm{m}^{3} \text { shown in (b). }
$$

For this synthetic test, first, we substitute the actual density value $m$ and the actual Gaussian noise $\Delta d$ into equation (17) to obtain the accurate $\lambda$ of the L-M method shown in Fig. 3, and $m_{i}$ is equal to a very tiny constant (e.g. $10^{-50}$ ) to replace 0 when $m_{i}=0$. We find that $\lambda$ fluctuates severely between about $-6 \times 10^{33} \sim 2 \times 10^{33}$ in Fig. 3 . 


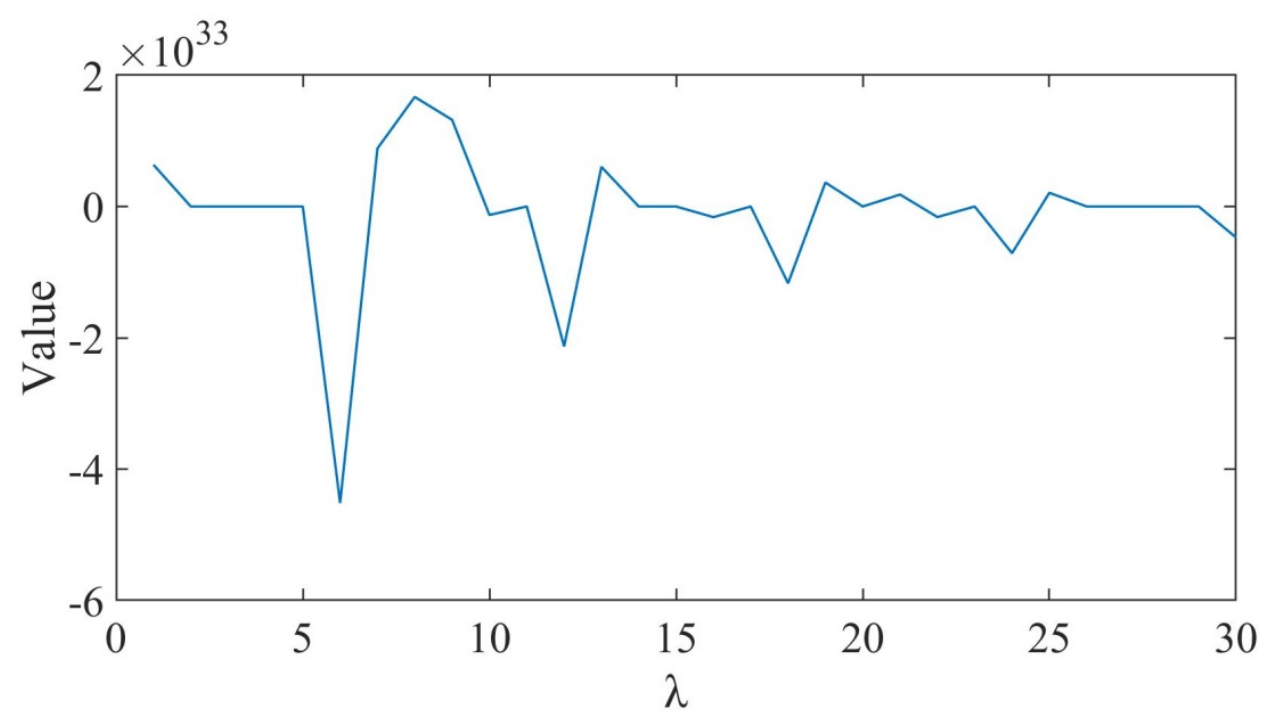

Fig. 3. The accurate value of $\lambda$ of the L-M method obtained by equation (17) using the actual density value $m$ and the actual Gaussian noise $\Delta d$.

Second, we substitute the accurate $\lambda$ shown in Fig. 3 into equation (18) and the inversion results is obtained by the L-M method shown in Fig. 4. We find that the inversion results are completely consistent with the real values $m$, which means the damping factor $\lambda$ by equation (17) is correct for obtaining the accurate inversion results.

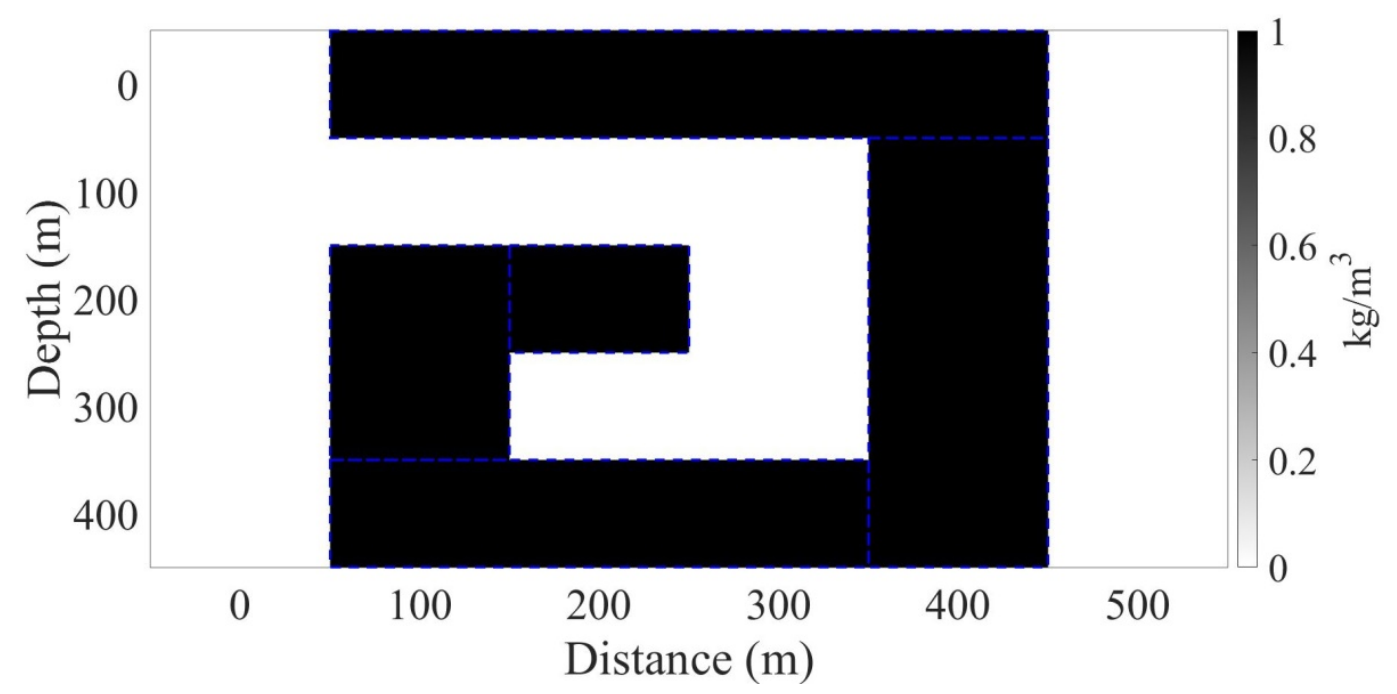

Fig. 4. The inversion results based on the accurate $\lambda$ shown in Fig. 3 obtained by the L-M

method. The inversion results are completely consistent with the real density value $m$.

Finally, according to conclusion i) and ii) and the synthetic test above, we summarize what the characteristics of the damping factor in the L-M method should have: the damping factor $\lambda_{0}$ in the L-M method should be replaced by a vector $\lambda$ (conclusion $\mathrm{i}$ ), in which $\lambda_{i}$ may be positive or 
negative and even a constant approaching $\infty$ (Fig. 3). This is very different from what we thought in the past that "the damping factor is just a tiny and positive constant".

\section{The modified L-M method based on the Binary inversion model}

The principle of the modified L-M method based on the Binary inversion model

In the previous section, on one hand, we find that the accurate damping factor $\lambda$ can be obtained by the real density value $m$ and the real Gaussian noise $\Delta d$, but neither of them can be known in the actual situation. Therefore, we must roughly estimate $\lambda$ in some way. On the other hand, $\lambda$ of the L-M method fluctuates severely as shown in Fig. 3 especially when there are density blocks with a value of 0 for the binary inversion model, the severe fluctuation of $\lambda$ leads to the difficulty of estimating $\lambda$ accurately. And this section attempts to modify the L-M method for the Binary inversion model so that the real damping factor $\lambda$ is stable and easy to estimate in the modified L-M method.

Actually, the constraint as shown in equation (4) of the L-M method is just a kind of loose constraint for the binary inversion model. Since there are only two kinds of values in the Binary inversion model as shown below:

$$
\widetilde{m}_{i}=m_{1} \text { or } m_{2}, i=1,2,3, \ldots, q
$$

Apparently, the tighter constraint can be imposed on equation (2), which is:

$$
\left(\widetilde{\boldsymbol{m}}_{i}-\boldsymbol{m}_{1}\right)\left(\widetilde{\boldsymbol{m}}_{i}-\boldsymbol{m}_{2}\right)=\mathbf{0}, i=\mathbf{1}, 2,3, \ldots, q
$$

Furthermore, the new form of gravity inversion equation is given as follows:

$$
\begin{gathered}
\text { Minimize }\left\|\mathbf{W}_{\mathbf{d}}(\mathbf{G} \widetilde{\boldsymbol{m}}-\widetilde{\boldsymbol{d}})\right\|_{2}^{2} \\
\text { s.t. }\left(\widetilde{\boldsymbol{m}}_{i}-\mathbf{m}_{1}\right)\left(\widetilde{\boldsymbol{m}}_{i}-\mathbf{m}_{2}\right)=\mathbf{0}, \boldsymbol{i}=\mathbf{1}, \mathbf{2}, 3, \ldots, \mathbf{q}
\end{gathered}
$$

Similar to the derivation of the equation (6), equation (22) derived from equation (21) has the following form (The only difference between derivation of equation 22 and equation 6 is the vector $\lambda$ is directly applied to the derivation of equation 22 instead of the constant $\lambda_{0}$ in equation 6):

$$
G^{\mathrm{T}} \mathbf{W G} \tilde{\boldsymbol{m}}_{i}+\operatorname{diag}(\lambda) \tilde{m}_{i}=\mathbf{G}^{\mathrm{T}} \mathbf{W} \widetilde{d}+\frac{1}{2}\left(\mathbf{m}_{1}+\mathbf{m}_{2}\right) \lambda,
$$




$$
\lambda=\left[\lambda_{1}, \lambda_{2}, \ldots, \lambda_{q}\right]^{\mathrm{T}} .
$$

Comparing to equation (6), the term $\frac{1}{2}\left(\mathrm{~m}_{1}+\mathrm{m}_{2}\right) \lambda$ is added to the right side of equation (22) besides the damping factor converting from $\lambda_{0}$ to the vector $\lambda$.

And the specific inversion solution is as follows:

$$
\widetilde{\boldsymbol{m}}=\left(\mathbf{G}^{\mathrm{T}} \mathbf{W G}+\operatorname{diag}(\lambda)\right)^{-1}\left[\mathbf{G}^{\mathrm{T}} \mathbf{W} \widetilde{\boldsymbol{d}}+\frac{1}{2}\left(\mathbf{m}_{1}+\mathbf{m}_{2}\right) \lambda\right] .
$$

Next, we still use the same analysis process of the L-M method (equation 8 14) to analyze the modified L-M method. We substitute equation (8) into equation (22), and if we want $\Delta m \rightarrow 0$, then:

$$
\begin{gathered}
\operatorname{diag}(\lambda)\left[2 m-\left(m_{1}+m_{2}\right) e\right] \approx 2 G^{\mathrm{T}} W \Delta d \\
e=[1,1, \ldots, 1]^{T}
\end{gathered}
$$

And for the $i$ th expression in equation (24), we have:

$$
\lambda_{i}\left[2 m_{i}-\left(\mathbf{m}_{1}+\mathbf{m}_{2}\right)\right] \approx 2 \mathbf{G}^{\mathrm{T}}{ }_{\mathrm{i}} \mathbf{W} \Delta d .
$$

And for the Binary inversion model, because $m_{i}=m_{1}$ or $m_{2}$, we substitute it into equation 25 and we obtain:

$$
\lambda_{i} \approx\left\{\begin{array}{cc}
\frac{2 \mathrm{G}^{\mathrm{T}}{ }_{\mathrm{i}} \mathrm{W} \Delta d}{m_{1}-m_{2}} & m_{i}=m_{1} \\
-\frac{2 \mathrm{G}_{\mathrm{i}} \mathrm{W} \Delta d}{m_{1}-m_{2}} & m_{i}=m_{2}
\end{array}\right.
$$

Synthetic test on the damping factor of the modified L-M method

We still use the inversion model and the corresponding gravity data shown in Fig. 2. The damping factor $\lambda$ is obtained by equation (26) shown in Fig. 5. Comparing to the precise value obtained by the L-M method shown in Fig. 3, the value of the precise value $\lambda$ in the modified LM method is more stable which ranges from $-1 \times 10^{-16}$ to $1.5 \times 10^{-16}$, whereas the precise value $\lambda$ in the L-M method fluctuates severely between about $-6 \times 10^{33} \sim 2 \times 10^{33}$ shown Fig. 3 . 


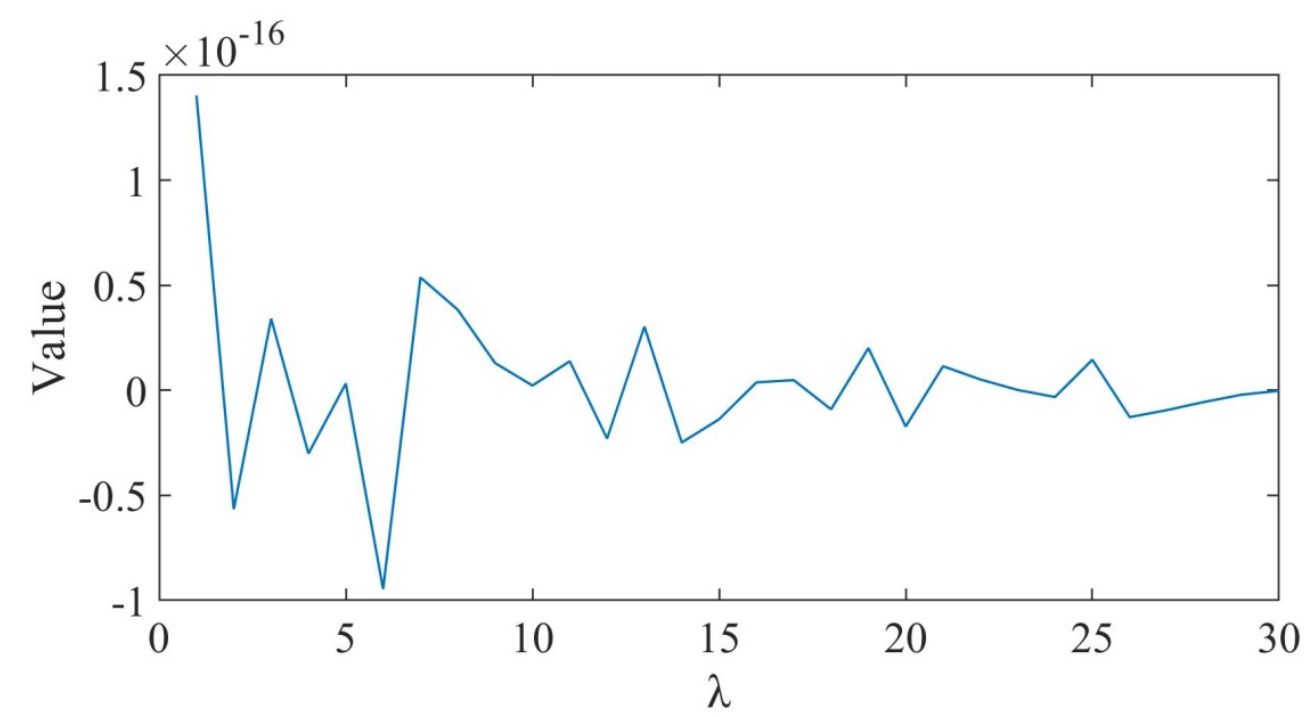

Fig. 5. The accurate value $\lambda$ of the modified L-M method obtained by equation (26). The values of $\lambda$ is very stable ranging from $-1 \times 10^{-16}$ to $1.5 \times 10^{-16}$.

We also calculate the inversion results $\tilde{m}$ shown in Fig. 6 using $\lambda$ obtained by the modified L-M method shown in Fig. 5 and $\tilde{m}$ is the same as the real values $m$.

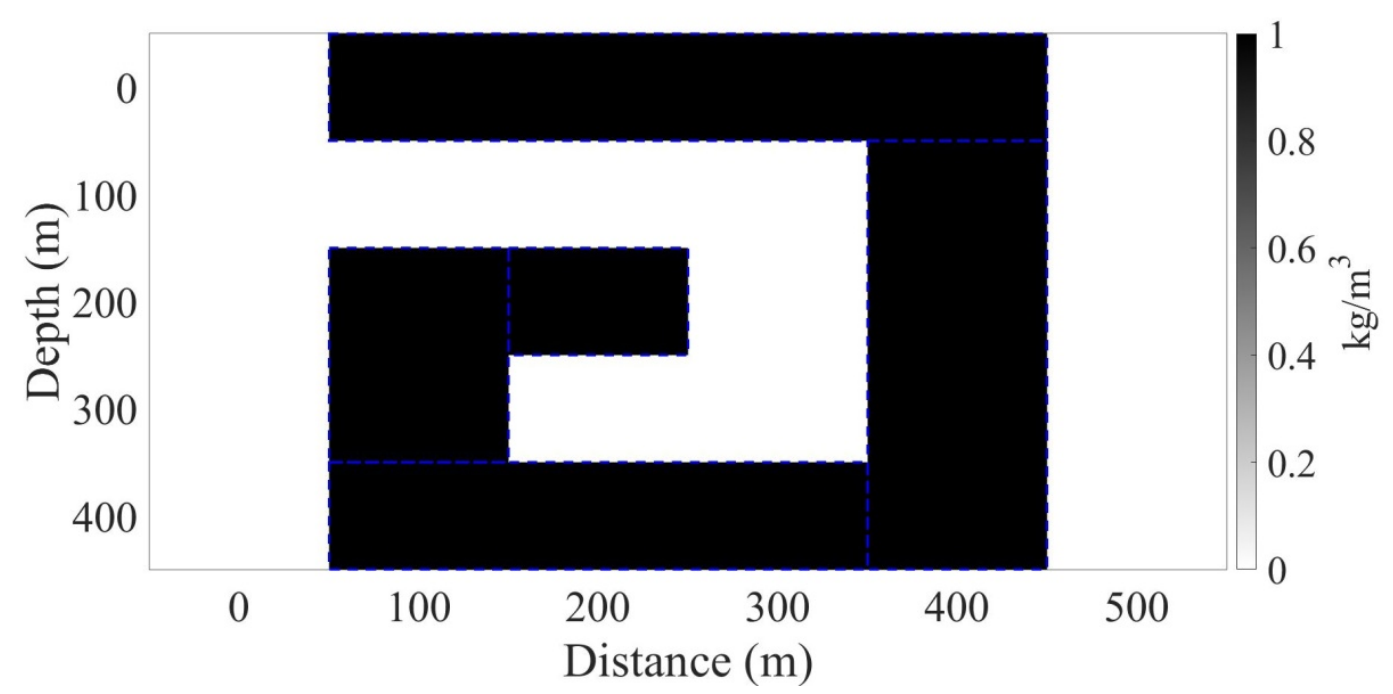

Fig. 6. The inversion results based on the precise $\lambda$ shown in Fig. 5 obtained by the modified

L-M method. The inversion results are completely consistent with the real density value $m$.

How to Estimate $\lambda$ accurately

The significant difference between the modified L-M method and the L-M method is the value of the damping factor of the modified L-M method is stable but the L-M method is not. Once the 
value of $\lambda$ is stable, to estimate $\lambda$ accurately is relatively easy. And we give the following estimation method:

$$
\tilde{\lambda}_{i}= \begin{cases}\frac{2 G^{T}{ }_{i} W \Delta \widetilde{d}}{m_{1}-m_{2}} & n_{m_{1}} \gg n_{m_{2}}(1) \\ -\frac{2 G^{T}{ }_{i} W \Delta \widetilde{d}}{m_{1}-m_{2}} & n_{m_{1}} \ll n_{m_{2}}(2) ; i=1,2,3, \ldots, \mathbf{q} . \\ \frac{2 G^{T}{ }_{i} W \Delta \widetilde{d}}{m_{1}-m_{2}} \tau & n_{m_{1}} \approx n_{m_{2}}(3) .\end{cases}
$$

In equation (27), $\tilde{\lambda}_{i}$ represents the estimation of $\lambda_{i} . \Delta \tilde{d}$ is the Gaussian noise with the same distribution as $\Delta d$ (we can use math software to produce $\Delta \tilde{d}$ easily). $n_{m_{1}}$ and $n_{m_{2}}$ represent the number of the density blocks equal to $\mathrm{m}_{1}$ and $\mathrm{m}_{2}$, respectively. $\tau$ is a constant equal to -1 or 1 (determined randomly).

Analysis of equation (27): According to equation (26), if $\mathrm{n}_{\mathrm{m}_{1}} \gg \mathrm{n}_{\mathrm{m}_{2}}$, the number of $\tilde{\lambda}_{i}$ equal to $\frac{2 \mathrm{G}_{\mathrm{T}} \mathrm{W} \Delta d}{m_{1}-m_{2}}$ is greater than $-\frac{2 \mathrm{G}^{\mathrm{T}} \mathrm{i} \Delta d}{m_{1}-m_{2}}$, and $\tilde{\lambda}_{i}$ should be equal to $\frac{2 \mathrm{G}^{\mathrm{T}} \mathrm{W} \Delta d}{m_{1}-m_{2}}$ as many as possible to ensure that as many as $\tilde{\lambda}_{i}$ is close to the actual value $\lambda_{i}$, and vice versa. However, if $\mathrm{n}_{\mathrm{m}_{1}} \approx \mathrm{n}_{\mathrm{m}_{2}}$, the number of $\tilde{\lambda}_{i}$ equal to $\frac{2 \mathrm{G}^{\mathrm{T}}{ }_{\mathrm{i}} \mathrm{W} \Delta \tilde{d}}{m_{1}-m_{2}}$ and $-\frac{2 \mathrm{G}_{\mathrm{T}}^{\mathrm{T}} \mathrm{W} \Delta \tilde{d}}{m_{1}-m_{2}}$ are basically the same, we can only introduce a random constant $\tau(-1$ or 1$)$ to randomly determine whether $\tilde{\lambda}_{i}$ is equal to $\frac{2 \mathrm{G}^{\mathrm{T}}{ }_{\mathrm{W}} \mathrm{W} \Delta \tilde{d}}{\mathrm{~m}_{1}-\mathrm{m}_{2}}$ or $-\frac{2 \mathrm{G}^{\mathrm{T}} \mathrm{W} \Delta \tilde{d}}{\mathrm{~m}_{1}-\mathrm{m}_{2}}$

For the inversion model shown in Fig. 2, the number of $m_{i}=m_{1}$ (16 blocks) are almost the same as $m_{i}=m_{2}$ (14 blocks), we use equation 27-3 to obtain $\tilde{\lambda}_{i}$. And the estimation $\tilde{\lambda}_{i}$ and the accurate $\lambda_{i}$ are drawn in the figure (Fig. 7), we can see that $\tilde{\lambda}_{i}$ and $\lambda_{i}$ are very close, the relative error obtained by equation 28 is only about $104.50 \%$ for the extremely tiny $\lambda_{i}$ (The maximum value does not exceed $\left.3 \times 10^{-16}\right)$.

$$
\text { Relative Error }=\frac{\sum_{i}\left|\tilde{\lambda}_{i}-\lambda_{i}\right|}{\sum_{i}\left|\lambda_{i}\right|}
$$




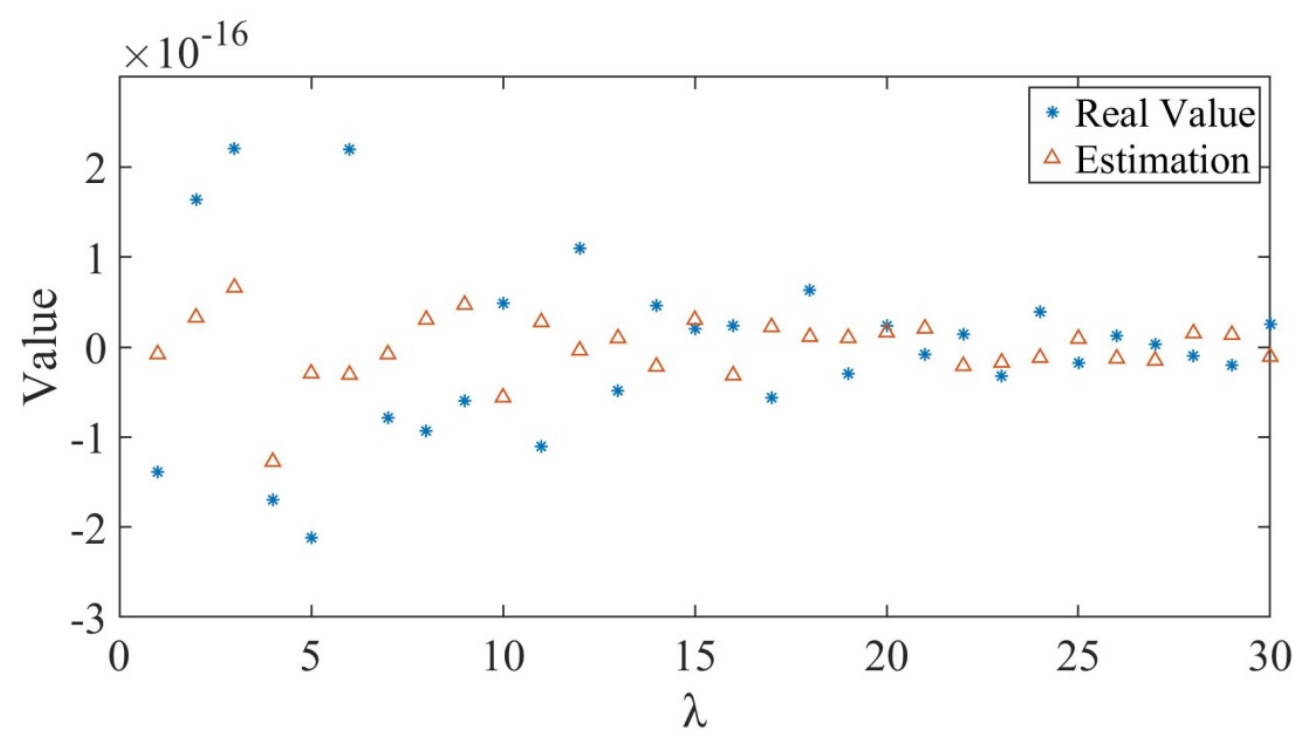

Fig. 7. Comparison of the real values $\lambda_{i}$ (asterisk) and the estimation solutions $\tilde{\lambda}_{i}$ (triangles)

However, $\tilde{\lambda}_{i}$ is very close to $\lambda_{i}$ shown in Fig. 7, the inversion results are still not ideal except that the shallowest layer of the inversion results is in good agreement with the actual situation. We use a new form of figure (Fig.8) to make it easier to observe the differences between the inversion results $\widetilde{m}$ and the real density values $m$. The abscissa in Fig.8 represents the density blocks arranged in the specified order corresponding to the order of the density blocks from left to right and top to bottom in Fig. 2, and the ordinate represents the value of the corresponding density block. In Fig. 8, the shallowest layer (the $1^{\text {st }}$ to $6^{\text {th }}$ density blocks) of the density blocks is indeed ideal but the others are not (the $7^{\text {th }}$ to $30^{\text {th }}$ density blocks).

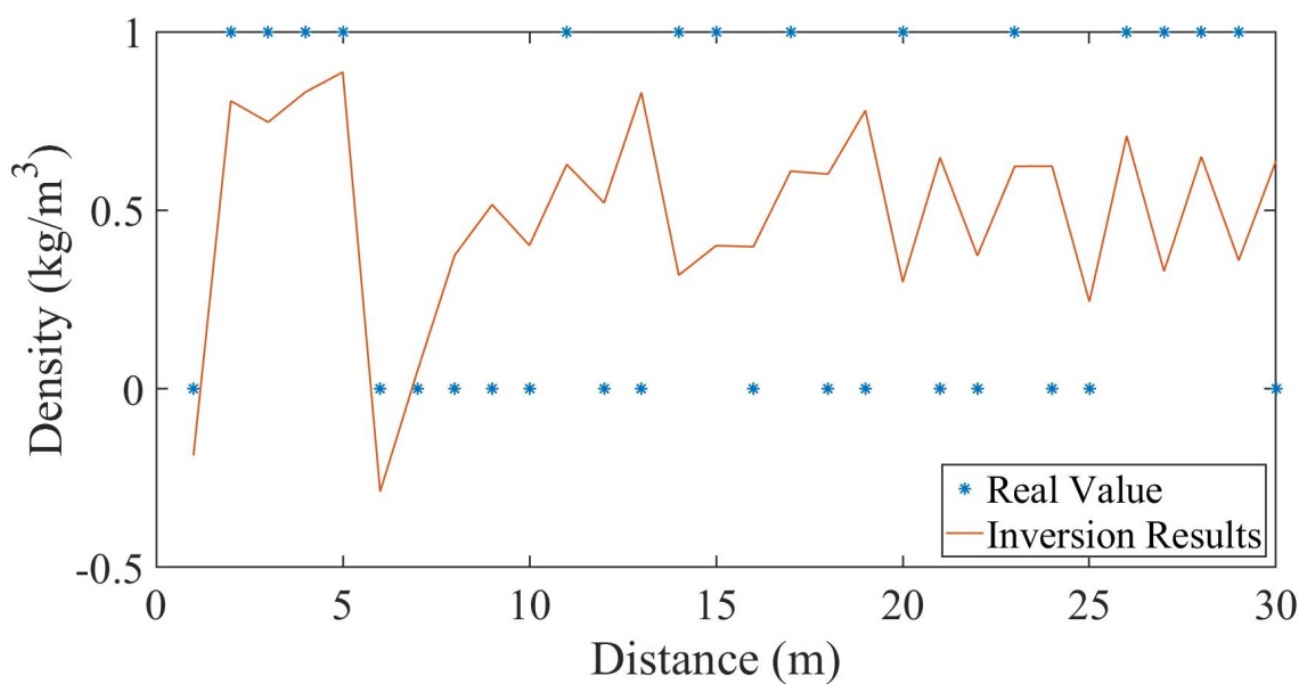


Fig. 8. Comparison of the real density values (asterisk) obtaind by the modified L-M method combined with the estimation $\tilde{\lambda}_{i}$ shown in Fig. 7 and the inversion results (solid line).The abscissa represents the sequence of the density blocks, this sequence corresponds to the density blocks shown in Figure 2 in the order from left to right and top to bottom.

\section{Conclusion}

This paper analyzes the inapplicability of only considering the damping factor in the L-M algorithm as a positive constant for obtaining ideal inversion results, and the actual characteristics of the damping factor of the L-M method are given: it should be a vector composed by the values which can be positive or negative and even a constant approaching $\infty$. This is very different from what we thought in the past in the geophysical inversion field that "the damping factor is just a tiny and positive constant". And due to the severe fluctuation of the actual values of the damping factor in the L-M method for the Binary inversion model, the modified L-M method is proposed and the actual value of the corresponding damping factor is more stable for facilitate estimation. And the specific strategy of estimating the actual damping factor of the modified L-M method for the binary inversion model is also proposed. However, the estimation is very close to the actual damping factor but the inversion results are still not ideal except the inversion results of the shallow layer.

1. Levenberg KQ. A Method for The Solution of Certain Non-Linear Problem in Least Squares. Quartaplmath 1944.

2. Marquardt DW. An algorithm for least-squares estimation of non-linear parameters. Journal of the Society for Industrial and Applied Mathematics 1963, 11(2): 431-441.

3. Last BJ, Kubik K. Compact gravity inversion. Geophysics 1983, 48: 713-721.

4. Li Y, Oldenburg DW. 3-D inversion of gravity data. Geophysics 1998, 63.

5. Mendona CA, Chaves C. Mass-constrained basin basement mapping. Geophysics 2021, 86(3). 
6. Camacho AG, Fernández J, Gottsmann J. A new gravity inversion method for multiple subhorizontal discontinuity interfaces and shallow basins. Journal of Geophysical Research: Solid Earth 2011, 116.

7. Barbosa VCF, Silva JBC. Generalized compact gravity inversion. Geophysics 1994, 59(1): 57-68.

8. Li Z, Yao C, Zheng Y, Zhang Y, Wang J. 3D magnetic sparse inversion using an interiorpoint method. Geophysics 2018, 83(3): 15-32.

9. Ghalehnoee MH, Ansari A, Ghorbani A. Improving compact gravity inversion using new weighting functions. Geophysical Journal International 2017, 208(2): 546-560.

10. Tian Y, Li H, Wang Y, Ye Q, Guo A. Gravity Gradient Inversion of Gravity Field and Steady-State Ocean Circulation Explorer Satellite Data for the Lithospheric Density Structure in the Qinghai-Tibet Plateau Region and the Surrounding Regions. Journal of Geophysical Research: Solid Earth 2021, 126.

11. Pilkington M. 3-D magnetic imaging using conjugate gradients. Geophysics 1997, 15(1): $1132-1142$.

12. Sun J, Li Y. Adaptive Lp inversion for simultaneous recovery of both blocky and smooth features in a geophysical model. Geophysical Journal International 2014, 197(2): 882-899.

13. Tikhonov AN, Arsenin VY. Solutions of ill-posed problems. W. H. Winston \& Sons, New York., 1977. 
14. Guillen A, Menichetti V. Gravity and magnetic inversion with minimization of a specific functional. Geophysics 1984, 49(8): 1354-1360.

15. Portniaguine O, Zhdanov MS. Focusing geophysical inversion images. Geophysics 1999, 64(3): $874-887$.

16. Camacho AG, Fernández J, Gottsmann J. The 3-D gravity inversion package GROWTH2.0 and its application to Tenerife Island, Spain. Computers \& Geosciences 2011, 37(4): 621-633.

17. Camacho AG, Montesinos FG, Vieira R. Gravity inversion by means of growing bodies. Geophysics 2000, 65(1): 95-101.

18. Camacho AG, Prieto JF, Aparicio A, Ancochea E, Fernández J. Upgraded GROWTH 3.0 software for structural gravity inversion and application to El Hierro (Canary Islands). Computers \& Geosciences 2021, 150. 